\title{
Is Scapular Stabilization Exercise Effective for Managing Nonspecific Chronic Neck Pain?: A Systematic Review
}

\author{
Yong Gon Seo ${ }^{1}$, Won Hah Park ${ }^{1}$, Chong Suh Lee ${ }^{1}$, Kyung Chung Kang ${ }^{2}$, \\ Kyoung Bin $\mathrm{Min}^{3}$, Sang Min Lee ${ }^{1}$, Jae Chul Yoo ${ }^{1}$ \\ ${ }^{1}$ Division of Sports Medicine, Department of Orthopedic Surgery, Samsung Medical Center, Sungkyunkwan University School of Medicine, Seoul, Korea \\ ${ }^{2}$ Department of Orthopedic Surgery, Kyung Hee University Hospital, Kyung Hee University School of Medicine, Seoul, Korea \\ ${ }^{3}$ Department of Sports Medicine, Korea University, Sejong, Korea
}

Scapular stabilization is thought to have an important role in improving pain and dysfunction around the neck and shoulders, but evidence of this is lacking. We aim to systematically review the effect of a scapular stabilization exercise (SSE) on pain and dysfunction in patients with nonspecific chronic neck pain (NP). We searched the PubMed, EMBASE, CINAHL, and Cochrane Library databases using the terms (NP [MeSH] OR NP OR cervical pain OR neck ache OR cervicalgia) AND (scapular exercise OR periscapular exercise OR SSEs). We included suitable studies that met the study's inclusion criteria. Among the 227 studies identified by our search strategy, a total of four (three randomized controlled studies and one prospective study) met the inclusion criteria. The SSE was intense. It included three sets of 10 repetitions. In most of the studies, the exercises were conducted 3 times per week. Most studies reported that the SSE improved pain and dysfunction in patients with nonspecific chronic NP; however, the reviewed articles did not use the same variables for measurement. Additionally, the sample size was small. Although several studies show that SSE might improve NP and dysfunction, the effects of SSE on pain and dysfunction in the neck region remain unclear because the number of studies was small. Further high-quality studies are necessary to identify the detailed effects of SSE in patients with NP.

Keywords: Neck pain; Exercise therapy; Scapula

\section{Introduction}

Neck pain (NP), a common musculoskeletal disorder, involves discomfort and pain around the neck region, resulting in limited neck function [1]. The annual prevalence of NP is reportedly $30 \%-50 \%$ in the adult population, and it persisted until the 1-year follow-up in $34.3 \%$ of patients who developed NP [1,2]. Persistent pain can reduce the quality of life (QOL) of patients, as well as cause an economic burden [3].

Various factors-such as female sex, older age, high job demands, low social/work support, ex-smoker status, and a history of lower back disorders-are linked to the development of nonspecific NP [4,5]. Among them, the working conditions of office workers cause postural malalignment in the neck and shoulder regions [6]. Patients with nonspecific NP display altered dynamic scapular stability during scapular orientation [7-9]. There is increasing interest in

Received Feb 19, 2019; Revised Mar 22, 2019; Accepted Apr 7, 2019

Corresponding author: Jae Chul Yoo

Department of Orthopedic Surgery, Samsung Medical Center, Sungkyunkwan University School of Medicine, 81 Irwon-ro, Gangnam-gu, Seoul 06351, Korea

Tel: +82-2-3410-3501, Fax: +82-2-3410-0061, E-mail: shoulderyoo@gmail.com 
scapular stabilization for patients with NP because of the close relationship between the neck and scapula [10].

The scapular bone connects the neck and shoulder and plays a very important role in stabilizing the neck and shoulder complex. A previous study reported that patients who suffer from NP have malfunctions, such as decreased clavicular retraction and upward rotation [8]. The axioscapular muscles, including the trapezius, serratus anterior, rhomboid major and minor, and levator scapulae, are attached to the scapular bone and can attribute to movement of the neck and shoulder complex [9]. Several studies demonstrated that tightness and weakness of the axioscapular muscles could induce scapular dyskinesis, which refers to abnormal scapular position or movement [11-13]. In recent years, several studies have examined scapular stabilization in patients with NP [14-17].

According to two systematic reviews $[18,19]$, general exercise therapy (strengthening and endurance exercises for the neck region) in patients with nonspecific NP was effective in treating NP and reducing functional disability. Despite the intervention of comprehensive exercise interventions in these patients, little is known about the effect of the scapula on cervical pain, neck function, and the QOL in patients with NP. Although several studies have reported that scapular-focused exercises reduce pain and improve function in shoulder disease [20-23], data on the effectiveness of scapular stabilization exercise (SSE) on cervical pain and QOL is lacking.

The goal of this systematic review is to identify the ef- fectiveness of SSE on function and pain in patients with nonspecific NP and suggest its importance in the treatment of cervical pain.

\section{Materials and Methods}

\section{Data sources and searches}

The PubMed, EMBASE, CINAHL, and Cochrane Library databases were searched using the following terms: (NP [MeSH] OR NP OR cervical pain OR neck ache OR cervicalgia) AND (scapular exercise OR periscapular exercise OR SSEs). The search included articles published between 1970 and 2018, and all articles identified in the search were evaluated.

\section{Inclusion criteria}

Prospective studies (nonrandomized) and randomized controlled trials with comparison trials were included. Reports conducted in humans aged $>18$ years with nonspecific NP were included. Only studies published in English were eligible for inclusion. Reviews and case reports were excluded. Studies that included subjects with a history of cervical disc herniation and cervical surgery were also excluded.

\section{Study selection}

Two authors (Seo and Min) independently reviewed the

Table 1. The PEDro scale score of the included studies

\begin{tabular}{|c|c|c|c|c|}
\hline Articles PEDro criteria & $\begin{array}{c}\text { Andersen et al. } \\
\text { [14] (2014) }\end{array}$ & $\begin{array}{l}\text { Im et al. [15] } \\
(2016)\end{array}$ & $\begin{array}{l}\text { Kang et al. [16] } \\
\text { (2018) }\end{array}$ & $\begin{array}{l}\text { Yidiz et al. [17] } \\
\text { (2018) }\end{array}$ \\
\hline Eligibility criteria & Yes & No & Yes & Yes \\
\hline Random allocation & Yes & Yes & No & Yes \\
\hline Concealed allocation & No & No & No & No \\
\hline Baseline similarity & Yes & Yes & Yes & Yes \\
\hline Blinding of subjects & No & No & No & No \\
\hline Blinding of therapists & No & No & No & No \\
\hline Blinding of assessors & Yes & No & No & Yes \\
\hline Measures of key outcomes from more than $85 \%$ of subjects & No & Yes & Yes & No \\
\hline Intention-to-treat analysis & Yes & No & No & No \\
\hline Between-groups statistical comparisons & Yes & No & No & Yes \\
\hline Point measures and measures of variability & Yes & Yes & Yes & Yes \\
\hline Total & 7 & 4 & 4 & 6 \\
\hline
\end{tabular}


studies identified by the search and extracted articles that met the inclusion criteria. The titles of all articles were reviewed first. Disagreement between the two reviewers was resolved by discussion. In cases in which an agreement could not be reached, a third author (Kang) reviewed the title to determine whether it should be included. The same procedure was used to review the abstracts and, again, to review the full text. All articles were rated using the PEDro scale (Table 1).

\section{Results}

\section{Study identification and characteristics}

The search strategy initially yielded 227 articles, of which four met our inclusion criteria. In the title review, 200 studies were excluded because of search term mismatch. Nine studies were excluded because of duplication, leav-

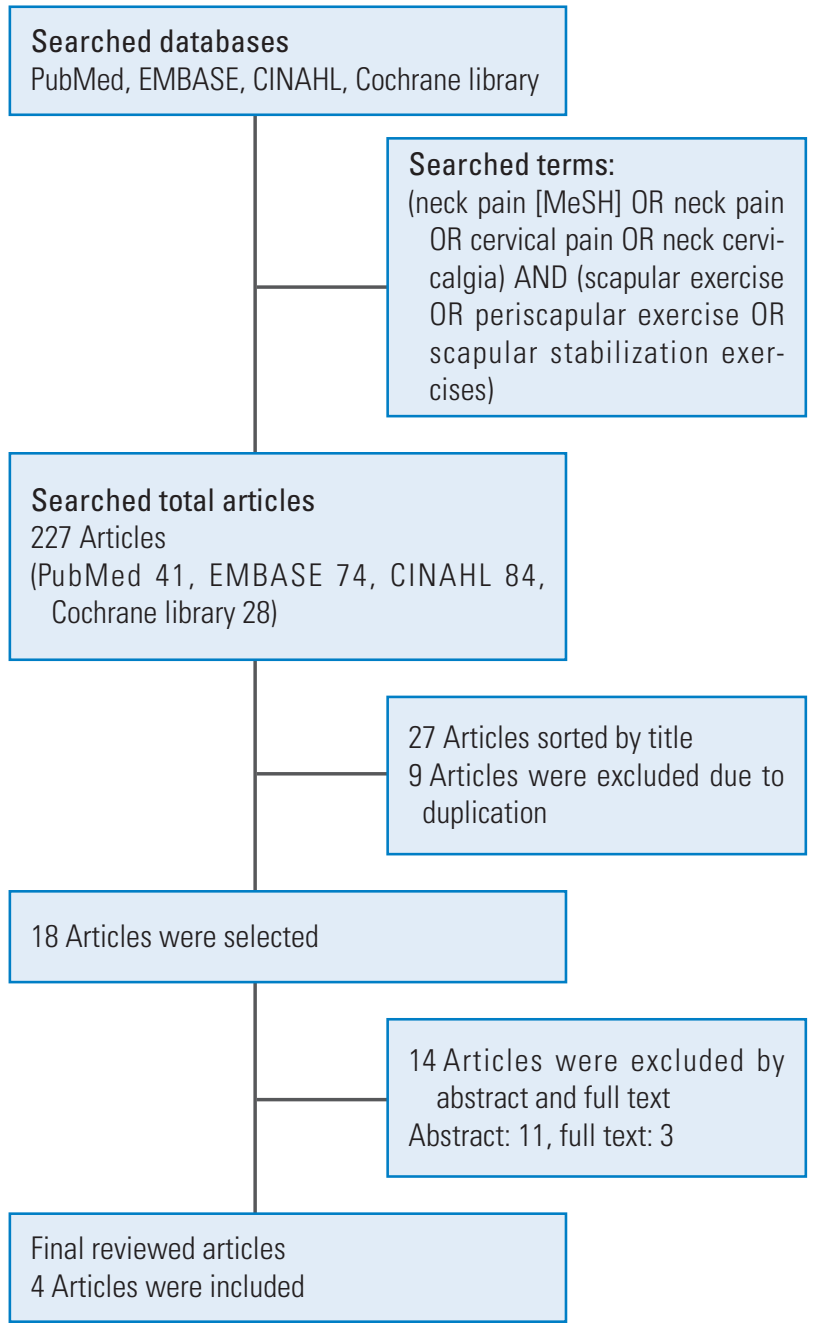

Fig. 1. Flowchart of articles search. ing 18 articles for abstract screening. Of them, 11 were excluded and seven met the inclusion criteria. Three articles were excluded by full text review, and the remaining four were included in this systematic review (Fig. 1). The included studies (three randomized controlled trials, one prospective study) were published between 2014 and 2018 in Korea, Denmark, and Turkey. Most studies defined chronic NP as pain that persisted for 3-6 months in the cervical and shoulder region. The definition of SSE was not described in all reviewed articles (Table 2).

\section{Scapular stabilization exercise}

The reviewed articles did not define SSE. Strengthening exercises were most often used. The upper trapezius and serratus anterior muscles were commonly targeted [1417]. The lower trapezius and sternocleidomastoid muscles were targeted in a study conducted by Kang et al. [16] The exercise types were varied. A total of 14 exercisesincluding press-up in a chain, push-up plus [14], supine deep breathing, supine shoulder at $90^{\circ}$ of flexion with scapular protraction, arm raise in the quadruped position, lateral arm raise with $2 \mathrm{~kg}$ dumbbells, posture education [15], prone I, prone $\mathrm{Y}$, prone T, prone $\mathrm{W}$ [16], scapular retraction, lateral pull-down and push-up plus exercise [17] - were performed in the reviewed articles. The pushup plus exercise was conducted in two studies [14,17]. There is currently no international agreement on the definition of SSE.

\section{Exercise prescription}

All articles included in this study suggested an exercise prescription that consisted of exercise frequency, intensity, time, and type. The general exercise frequency was 3 times per week. However, one study prescribed a once-daily frequency [17]. The exercise intensity was described as repetitions, sets, and holding time. Commonly, 10-20 repetitions and three to five sets held for 3-10 seconds were performed. However, the exercise intensities progressively increased over the intervention period in only one study [17]. The maximal intervention time was 30 minutes in two studies [15,16] and 20 minutes in one study [14]. The study by Yidiz et al. [17] did not specify the exercise time. The exercise focused on stabilizing the scapular bone using strengthening exercise. The intervention duration in three of the studies was 4 weeks, whereas that of the 
Table 2. Summary of study characteristics and findings for reviewed studies about the scapular stabilization exercise

\begin{tabular}{|c|c|c|c|}
\hline Reference & Study deign and participants & Exercise intervention & Outcome and key finding \\
\hline Andersen et al. [14] (2014) & $\begin{array}{l}\mathrm{RCT}(\mathrm{N}=47) \\
\mathrm{n}=24, \text { training }(44 \pm 13 \text {; male } 5 \text {, } \\
\quad \text { female } 19) \\
\mathrm{n}=23 \text {, control }(45 \pm 11 \text {; male } 5, \\
\quad \text { female } 18)\end{array}$ & $\begin{array}{l}\text { F: } 3 \text { times/wk for } 10 \text { wk } \\
\text { I: } 10-20 \text { Rep/3-5 sets/10 sec hold } \\
\text { T: } 20 \text { min } \\
\text { T: Strengthening }\end{array}$ & $\begin{array}{l}\text { Self-rated pain intensity: decreased }(p<0.01) \\
\text { Pressure pain threshold: upper trapezius } \\
\text { increased from } 227 \text { to } 405 \mathrm{kPa}(p<0.05) \\
\text { Shoulder elevation strength increased in } 7.7 \\
\quad \mathrm{~kg}(p<0.01)\end{array}$ \\
\hline Im et al. [15] (2016) & $\begin{array}{l}\text { Prospective study }(\mathrm{N}=15) \\
n=8, \text { study }(35.5 \pm 8.8) \\
n=7 \text {, control }(35.7 \pm 9.8)\end{array}$ & $\begin{array}{l}\text { F: } 3 \text { times/wk for } 4 \text { wk } \\
\text { I: } 10 \text { Rep/3 sets } / 10 \mathrm{sec} \text { hold } \\
\text { T: } 30 \text { min } \\
\text { T: Strengthening }\end{array}$ & $\begin{array}{l}\text { ROM: CVA increased from } 38.2^{\circ} \pm 2.5^{\circ} \text { to } \\
49.3^{\circ} \pm 4.9^{\circ} \\
\text { Muscles EMG: UTM decreased from } \\
40.6 \% \pm 10.5 \% \text { to } 29.0 \% \pm 7.5 \% \text { and } \\
\text { SAM increased from } 28.5 \% \pm 7.6 \% \text { to } \\
37.4 \% \pm 8.1 \% \\
\text { VAS decreased from } 6.3 \pm 1.7 \text { to } 3.1 \pm 1.1 \text { and } \\
\text { NDI improved from } 14.4 \pm 8.1 \text { to } 7.9 \pm 3.1 \\
\text { WHO0OL-BREF improved from } 84.1 \pm 9.4 \text { to } \\
96.1 \pm 5.4\end{array}$ \\
\hline Kang et al. [16] (2018) & $\begin{array}{l}\text { RCT }(N=30) \\
n=15, \text { SSE }(31.8 \pm 7.5) \\
n=15, \operatorname{NSE}(33.8 \pm 4.8)\end{array}$ & $\begin{array}{l}\text { F: } 3 \text { times/wk for } 4 \text { wk } \\
\text { I: } 10 \text { Rep/3 sets/3 sec hold } \\
\text { T: } 30 \text { min } \\
\text { T: Strengthening }\end{array}$ & $\begin{array}{l}\text { ROM: CVA increased from } 49.5^{\circ} \pm 3.8^{\circ} \text { to } \\
53.9^{\circ} \pm 3.4^{\circ} \text { and CRA decreased from } \\
147.5^{\circ} \pm 3.7^{\circ} \text { to } 53.9^{\circ} \pm 3.4^{\circ} \\
\text { Muscles EMG: SCM and UTM decreased } \\
\text { from } 38.9 \% \pm 10.2 \% \text { to } 36.1 \% \pm 10.1 \% \\
\text { and from } 46.9 \% \pm 5.5 \% \text { to } 43.9 \% \pm 6.1 \% \text {, } \\
\text { respectively. } \\
\text { LTM and SAM increased from } 32.8 \% \pm 7.5 \% \\
\text { to } 36.8 \% \pm 9.1 \% \text { and from } 13.5 \% \pm 3.1 \% \text { to } \\
17.2 \% \pm 4.1 \% \text {, respectively }\end{array}$ \\
\hline Yidiz et al. [17] (2018) & $\begin{array}{l}\mathrm{RCT}(\mathrm{N}=30) \\
\mathrm{n}=15, \text { training }(32.8 \pm 7.4) \\
\mathrm{n}=15 \text {, control }(27.8 \pm 8)\end{array}$ & $\begin{array}{l}\text { F: Once a day for } 6 \text { wk } \\
\text { I: } 10 \text { Rep/2-3 sets } \\
\text { T: Non-mentioned } \\
\text { T: Strengthening }\end{array}$ & $\begin{array}{l}\text { VAS decreased and NDI: no difference } \\
\text { between groups ( } F=2.9, p=0.1) \\
\text { For scapular kinematics: no different be- } \\
\text { tween groups at } 30^{\circ}, 60^{\circ}, 90^{\circ} \text {, and } 120^{\circ}\end{array}$ \\
\hline
\end{tabular}

RCT, randomly controlled trial; Rep, repetition; ROM, range of motion; CVA, craniovertebral angle; EMG, electromyography; UTM, upper trapezius muscle; SAM, serratus anterior muscle; VAS, Visual Analog Scale; NDI, Neck Disability Index; WHOOOL-BREF, World Health Organization Quality of Life Assessment-Brief; SSE, scapular stabilization exercise; NSE, neck stabilization exercise; CRA, cranial rotation angle; SCM, sternocleidomastoid muscle; LTM, lower trapezius muscle.

fourth study was only 10 weeks [17]. The exercise intervention was conducted in a center with supervision.

\section{Clinical outcome}

1) Pain scale

A neck function scale score was included as a clinical outcome before and after the exercise intervention. The Visual Analog Scale (VAS) score was used to evaluate cervical pain in two articles $[15,17]$. The mean scores were $6.3 \pm 1.7$ before and $3.1 \pm 1.1$ after the intervention in one study [15], whereas the pain scores were not reported in the other study. One study used self-rated pain intensity for the study and showed significant intergroup change in the neck and shoulder regions $(p<0.01)$ [14]. The study conducted by Kang et al. [16] did not measure pain in the neck region.

\section{2) Neck function}

In two articles, neck function was evaluated using the Neck Disability Index (NDI) [15,17]. The NDI results were controversial between the two articles. One study published in 2016 described the change in NDI after exercise therapy as improvement from $14.4 \pm 8.1$ to $7.9 \pm 3.1$ in the study group [15]. Other studies showed a significant difference in the exercise group before and after intervention, but not between groups $(F=2.9, p=0.1)$. Two studies did not measure neck function after the intervention $[14,15]$.

3) Muscle activity

Muscle strength was assessed using an isometric exercise 
type in two articles $[14,16]$. The upper trapezius, lower trapezius, serratus anterior, and sternocleidomastoid muscles were targeted. The upper trapezius and serratus anterior muscles were selected in three studies $[14,16]$. The muscle activity results demonstrated decreased upper trapezius and increased serratus anterior and lower trapezius muscles activity after the intervention.

\section{4) Quality of life}

QOL was measured in only one study [15], which used the World Health Organization QOL Assessment-Brief questionnaire. QOL was improved after application of the SSE, with an increase from $84.1 \pm 9.4$ to $96.1 \pm 5.4$ in the exercise group.

\section{Discussion}

Here, we reviewed the recent literature to identify the effect of SSE on pain and the clinical outcome of patients with chronic NP. The exercise interventions mainly consisted of strengthening exercises, focusing on the periscapular muscles, including the trapezius, serratus anterior, and rhomboid muscles. Scapular stabilization may improve NP and function, but the evidence of this in the reviewed articles was insufficient.

\section{Scapular stabilization exercise}

The general program for patients with NP consists of stretching, strengthening, and functional exercises [19]. In this systematic review, all four studies used strengthening exercises for the periscapular muscles. One article mentioned that scapular stabilization involves a set of exercises that strengthen the shoulder girdle muscles to restore normal scapular motion and correct dyskinesia [24], but SSE was not described in any of the reviewed studies. We used the terms SSE, periscapular exercise, and scapular exercise to search for relevant articles. These terms were used differently in the previous studies. The serratus anterior, rhomboid major and minor, levator scapulae, and trapezius muscles are the main muscles that stabilize the scapula [24]. The scapula-stabilizing muscles were targeted for strengthening in the reviewed articles $[24,25]$. The exercise terminology differed among the reviewed articles, whereas three studies used specialized exercise terms, such as prone I, $\mathrm{Y}$, and $\mathrm{T}$. The fourth study used descriptive terms, such as supine shoulder at $90^{\circ}$ of flexion with scapular protraction, to describe the exercise [15]. Previous studies inconsistently used exercise terms. Therefore, further research using consistent terminology is needed to establish a definition of SSE [14-17].

\section{Exercise prescription}

All four reviewed articles suggested an exercise prescription according to exercise frequency, intensity, time, and type. According to a systematic review and meta-analysis [19], the exercise frequency for patients with chronic nonspecific NP was most commonly 3 times per week. In patients with shoulder disease, SSE was also performed 3 times per week $[21,22]$. The exercise frequency of 3 times per week in this systematic study was similar to that in previous studies $[19,21,22]$. The exercise intensity in the reviewed articles was described in terms of repetition and sets. In this study, repetition and sets were 10-20 and three to five, respectively. The exercise intensity with a low load and high repetition is associated with improved muscle endurance [26]. The scapular stabilizers are local muscles that contribute to stability of the scapular girdle. High repetition and multiple sets would improve muscle endurance. The progressive increase in intensity should be considered since not all articles in this study increased the exercise intensity over the intervention period. Exercise time is also an important factor in increasing the effect on exercise intervention, and the exercise times used in the reviewed articles were similar to those in previous studies $[21,22]$. Three studies included strengthening exercises using the patient's body weight, whereas the fourth study [16] used an exercise ball for stabilizing the scapular girdle. Several studies [21-23,27] reported SSE that consisted of stretching, strengthening, and functional exercise, although these studies focused on patients with shoulder disease. Further studies are needed to identify the effect of combined exercise types, including stretching or functional exercises, in patients with nonspecific NP for comparison with the results of other studies.

\section{Clinical outcome}

The incidence of self-reported NP was 213 per 1,000 persons, whereas the prevalence of pain for 12 months was $30 \%-50 \%$ [1]. NP relief is associated with increased functions and QOL in patients suffering from NP [19]. The VAS and Numeric Rating Scale are generally used to eval- 
uate pain level before versus after the intervention. The VAS and self-rated pain scale were used in the reviewed articles. All except one study reported that the pain was significantly changed after the intervention [16]. In other words, scapular stabilization may reduce NP. However, these studies had short-term intervention periods of 4-10 weeks; therefore, we could not identify the long-term effect of scapular stabilization. A systematic review reported that most previous studies included a short-term followup [16]. Therefore, further studies are required to identify the long-term effect and compare neck-focused intervention and SSE in patients with nonspecific NP.

Neck dysfunction is most commonly assessed using the NDI. In the reviewed articles, neck function was checked using the NDI in two articles $[15,17]$, whereas two others did not measure neck function before versus after the intervention $[14,16]$. Cervical dysfunction was improved in the intervention group in two studies. However, one study reported a significant difference within the exercise group, but not between the two groups [17]. Considering this review study, it is difficult to say that scapular stabilization improves neck dysfunction, although one study reported improvement [15]. Therefore, further studies are needed to identify the effect of SSE on neck function and to explain the mechanism by which neck function improves after scapular stabilization.

The trapezius muscles (i.e., upper and lower trapezius) and serratus anterior muscles, the main stabilizers, were targeted in this study [24]. The normal motion of the scapula is associated with proper firing and recruitment of scapula stabilizers involving the serratus anterior and the upper, middle, and lower trapezius muscles, resulting in the normal mechanism of force couples between muscles $[28,29]$. Muscular balance of the scapular stabilizers is among the important factors of proper neuromuscular control of the scapular motion. An altered scapular position (protracted and downwardly rotated) is commonly seen in patients with cervical pain [27]. This alteration is related to abnormal muscle function of the scapula stabilizer during arm movement, resulting in lower activity of the lower trapezius and a delayed onset and duration of serratus anterior activity $[11,28,30,31]$. To ensure normal muscle function during scapular motion, the main intervention goal is to inhibit over-activation of the upper trapezius and to enhance the weakened lower trapezius and serratus anterior muscles [9,27]. In the reviewed articles, the exercises focused on enhancing the periscapular muscles, such as the serratus anterior and the middle and lower trapezius muscles. An intervention consisting of different exercise types should be considered when analyzing the SSE results, although these exercises focused on the same muscles mentioned in previous studies.

QOL was evaluated in only one study using the QOL Assessment-Brief questionnaire [15]. QOL improved after a 4-week intervention. Various questionnaires have been used to evaluate QOL in patients with cervical pain [32]. Chronic NP is weakly associated with a decreased QOL as measured using the Short-Form 36 questionnaire $[33,34]$. Questionnaires are generally divided into two different types: disease-specific and general. Of the reviewed articles, only one measured QOL using a general questionnaire and the QOL Assessment-Brief questionnaire. Although one study reported SSE that could improve the QOL of patients with NP, the data is insufficient to allow the generalization of this result regarding the effect of SSE. Therefore, further studies are needed to identify the effect of SSE on QOL using disease-specific and general questionnaires.

The review study has some limitations. First, we searched only four large databases (PubMed, EMBASE, CINAHL, and Cochrane Library). This could have led to the occurrence of unidentified relevant articles. Further systematic reviews or meta-analyses that include searches of other large databases, such as OVID or Google, are required. Second, the reviewed articles provide relatively few data regarding the effect of SSE. This limitation could affect the generalization of the findings of this review study. Further large trials are needed to define SSE in patients with nonspecific NP considering the insufficiency of data from the four studies reviewed here. Third, the reviewed articles did not measure consistent variables, including pain scale, dysfunction, QOL, and testing methods. Therefore, it is difficult to generalize our results because of a mismatch of parameters, such as pain, dysfunction, and QOL. To overcome these problems, additional studies are needed to identify the clinical outcomes of SSE use in patients with nonspecific NP.

\section{Conclusions}

SSE could be considered an effective intervention for patients with nonspecific NP. However, it is difficult to generalize the results of this review study because the measured variables were not consistent among the four 
reviewed articles. Further studies are needed to gather additional strong evidence to identify the effectiveness of SSE for pain, dysfunction, and QOL of patients with NP.

\section{Conflict of Interest}

No potential conflict of interest relevant to this article was reported.

\section{References}

1. Hogg-Johnson S, van der Velde G, Carroll LJ, et al. The burden and determinants of neck pain in the general population: results of the Bone and Joint Decade 2000-2010 Task Force on Neck Pain and Its Associated Disorders. Spine (Phila Pa 1976) 2008;33(4 Suppl):S39-51.

2. Sadeghian F, Raei M, Amiri M. Persistent of neck/ shoulder pain among computer office workers with specific attention to pain expectation, somatization tendency, and beliefs. Int J Prev Med 2014;5:1169-77.

3. Haldeman S, Carroll L, Cassidy JD. Findings from the bone and Joint Decade 2000 to 2010 Task Force on Neck Pain and Its Associated Disorders. J Occup Environ Med 2010;52:424-7.

4. McLean SM, May S, Klaber-Moffett J, Sharp DM, Gardiner E. Risk factors for the onset of non-specific neck pain: a systematic review. J Epidemiol Community Health 2010;64:565-72.

5. Guzman J, Hurwitz EL, Carroll LJ, et al. A new conceptual model of neck pain: linking onset, course, and care: the Bone and Joint Decade 2000-2010 Task Force on Neck Pain and Its Associated Disorders. J Manipulative Physiol Ther 2009;32(2 Suppl):S17-28.

6. Mahmud N, Bahari SF, Zainudin NF. Psychosocial and ergonomics risk factors related to neck, shoulder and back complaints among Malaysia office workers. Int J Soc Sci Humanit 2014;4:260-3.

7. Helgadottir H, Kristjansson E, Mottram S, Karduna A, Jonsson $\mathrm{H}$ Jr. Altered alignment of the shoulder girdle and cervical spine in patients with insidious onset neck pain and whiplash-associated disorder. J Appl Biomech 2011;27:181-91.

8. Helgadottir H, Kristjansson E, Mottram S, Karduna AR, Jonsson H Jr. Altered scapular orientation during arm elevation in patients with insidious onset neck pain and whiplash-associated disorder. J Orthop
Sports Phys Ther 2010;40:784-91.

9. Cagnie B, Struyf F, Cools A, Castelein B, Danneels L, O'leary S. The relevance of scapular dysfunction in neck pain: a brief commentary. J Orthop Sports Phys Ther 2014;44:435-9.

10. Lluch E, Arguisuelas MD, Calvente Quesada O, et al. Immediate effects of active versus passive scapular correction on pain and pressure pain threshold in patients with chronic neck pain. J Manipulative Physiol Ther 2014;37:660-6.

11. Zakharova-Luneva E, Jull G, Johnston V, O'Leary S. Altered trapezius muscle behavior in individuals with neck pain and clinical signs of scapular dysfunction. J Manipulative Physiol Ther 2012;35:346-53.

12. Castelein B, Cools A, Bostyn E, Delemarre J, Lemahieu T, Cagnie B. Analysis of scapular muscle EMG activity in patients with idiopathic neck pain: a systematic review. J Electromyogr Kinesiol 2015;25:37186.

13. Behrsin JF, Maguire K. Levator scapulae action during shoulder movement: a possible mechanism for shoulder pain of cervical origin. Aust J Physiother 1986;32:101-6.

14. Andersen CH, Andersen LL, Zebis MK, Sjogaard G. Effect of scapular function training on chronic pain in the neck/shoulder region: a randomized controlled trial. J Occup Rehabil 2014;24:316-24.

15. Im B, Kim Y, Chung Y, Hwang S. Effects of scapular stabilization exercise on neck posture and muscle activation in individuals with neck pain and forward head posture. J Phys Ther Sci 2016;28:951-5.

16. Kang JI, Choi HH, Jeong DK, Choi H, Moon YJ, Park JS. Effect of scapular stabilization exercise on neck alignment and muscle activity in patients with forward head posture. J Phys Ther Sci 2018;30:804-8.

17. Yildiz TI, Turgut E, Duzgun I. Neck and scapulafocused exercise training on patients with nonspecific neck pain: a randomized controlled trial. J Sport Rehabil 2018;27:403-12.

18. Sihawong R, Janwantanakul P, Sitthipornvorakul E, Pensri P. Exercise therapy for office workers with nonspecific neck pain: a systematic review. J Manipulative Physiol Ther 2011;34:62-71.

19. Bertozzi L, Gardenghi I, Turoni F, et al. Effect of therapeutic exercise on pain and disability in the management of chronic nonspecific neck pain: systematic review and meta-analysis of randomized trials. Phys 
Ther 2013;93:1026-36.

20. Mulligan EP, Huang M, Dickson T, Khazzam M. The effect of axioscapular and rotator cuff exercise training sequence in patients with subacromial impingement syndrome: a randomized crossover trial. Int J Sports Phys Ther 2016;11:94-107.

21. Moezy A, Sepehrifar S, Solaymani Dodaran M. The effects of scapular stabilization based exercise therapy on pain, posture, flexibility and shoulder mobility in patients with shoulder impingement syndrome: a controlled randomized clinical trial. Med J Islam Repub Iran 2014;28:87.

22. Başkurt Z, Başkurt F, Gelecek N, Ozkan MH. The effectiveness of scapular stabilization exercise in the patients with subacromial impingement syndrome. J Back Musculoskelet Rehabil 2011;24:173-9.

23. Turgut E, Duzgun I, Baltaci G. Effects of scapular stabilization exercise training on scapular kinematics, disability, and pain in subacromial impingement: a randomized controlled trial. Arch Phys Med Rehabil 2017;98:1915-23.

24. Paine R, Voight ML. The role of the scapula. Int J Sports Phys Ther 2013;8:617-29.

25. Schory A, Bidinger E, Wolf J, Murray L. A systematic review of the exercises that produce optimal muscle ratios of the scapular stabilizers in normal shoulders. Int J Sports Phys Ther 2016;11:321-36.

26. Mangine GT, Hoffman JR, Gonzalez AM, et al. The effect of training volume and intensity on improvements in muscular strength and size in resistancetrained men. Physiol Rep 2015;3.
27. Cools AM, Struyf F, De Mey K, Maenhout A, Castelein B, Cagnie B. Rehabilitation of scapular dyskinesis: from the office worker to the elite overhead athlete. Br J Sports Med 2014;48:692-7.

28. Wegner S, Jull G, O'Leary S, Johnston V. The effect of a scapular postural correction strategy on trapezius activity in patients with neck pain. Man Ther 2010;15:562-6.

29. Lindstrom R, Schomacher J, Farina D, Rechter L, Falla D. Association between neck muscle coactivation, pain, and strength in women with neck pain. Man Ther 2011;16:80-6.

30. Sheard B, Elliott J, Cagnie B, O'Leary S. Evaluating serratus anterior muscle function in neck pain using muscle functional magnetic resonance imaging. J Manipulative Physiol Ther 2012;35:629-35.

31. Helgadottir H, Kristjansson E, Einarsson E, Karduna $\mathrm{A}$, Jonsson $\mathrm{H}$ Jr. Altered activity of the serratus anterior during unilateral arm elevation in patients with cervical disorders. J Electromyogr Kinesiol 2011;21:947-53.

32. Misailidou V, Malliou P, Beneka A, Karagiannidis A, Godolias G. Assessment of patients with neck pain: a review of definitions, selection criteria, and measurement tools. J Chiropr Med 2010;9:49-59.

33. Rezai M, Cote P, Cassidy JD, Carroll L. The association between prevalent neck pain and health-related quality of life: a cross-sectional analysis. Eur Spine J 2009;18:371-81.

34. Gurav RS, Nayak NK, Jain K. Health related quality of life in adults with neck pain: a cross sectional survey. Int J Health Sci Res 2013;3:53-7. 